\title{
Gold Catalysts Open a New General Chemoselective Route to Synthesize Oximes by Hydrogenation of $\alpha, \beta$-Unsaturated Nitrocompounds with $\mathbf{H}_{2}$
}

\section{SUPPORTING INFORMATION}

\section{Experimental Section}

\section{Catalyst Preparation}

The gold catalyst used in the present work consists on $1.5 \mathrm{wt} \%$ gold on $\mathrm{TiO}_{2}$, which can be supplied by the World Gold Council (reference catalysts, Type A) or, otherwise, could be prepared by depositing the gold from an aqueous solution of $\mathrm{HAuCl}_{4}$ on a sample of $\mathrm{TiO}_{2}$ (P25 Degussa). The deposition-precipitation procedure is done at $343 \mathrm{~K}$ and $\mathrm{pH}$ of 7, using $\mathrm{NaOH}$ to maintain this level during $2 \mathrm{~h}$. Under these conditions, a $80 \%$ in the efficiency of gold deposition is expected. The catalyst is then recovered, filtered, washed with deionized water, and dried at $373 \mathrm{~K}$ overnight. Finally, the powder is calcined at $673 \mathrm{~K}$ in air flow for 4 h. Under this procedure, $3.5 \mathrm{~nm}$ gold nanoparticles (averaged size) supported on $\mathrm{TiO}_{2}$ are obtained, as shown in Figure S1.

\section{Catalytic Experiments}

We have performed the catalytic experiments in a SPR16 reactor from Amtec (see Figure S2), which consist on a multiautoclave able to carry out up to sixteen batch reactions in parallel at high pressures (150 bar maximum). Firstly, the reaction mixture and an appropriate amount of catalyst are introduced into each autoclave (see Table S1). Then, a cold purge with $\mathrm{H}_{2}$ at room temperature is performed twice in order to completely evacuate the oxygen from the reactor. Before pressurizing the autoclave with $\mathrm{H}_{2}$, the reactor is heated up to the temperature set point (see Table 1 for temperature and pressure values), while stirring at 500 rpm. Once the reaction is completed, the autoclave is cooled down, depressurized, and its content transferred into a vial. Most of these actions, such as feeding reactants, controlling the reaction conditions, and taking samples from reactors can be automatically managed through the SPR16 control software. Products of the reactions can be analyzed by GC-MS after removing the catalyst from the reaction mixture. 
Figure S1. TEM image of the $1.5 \mathrm{wt} \% \mathrm{Au} / \mathrm{TiO}_{2}$ sample (a), and particles size distribution (b)
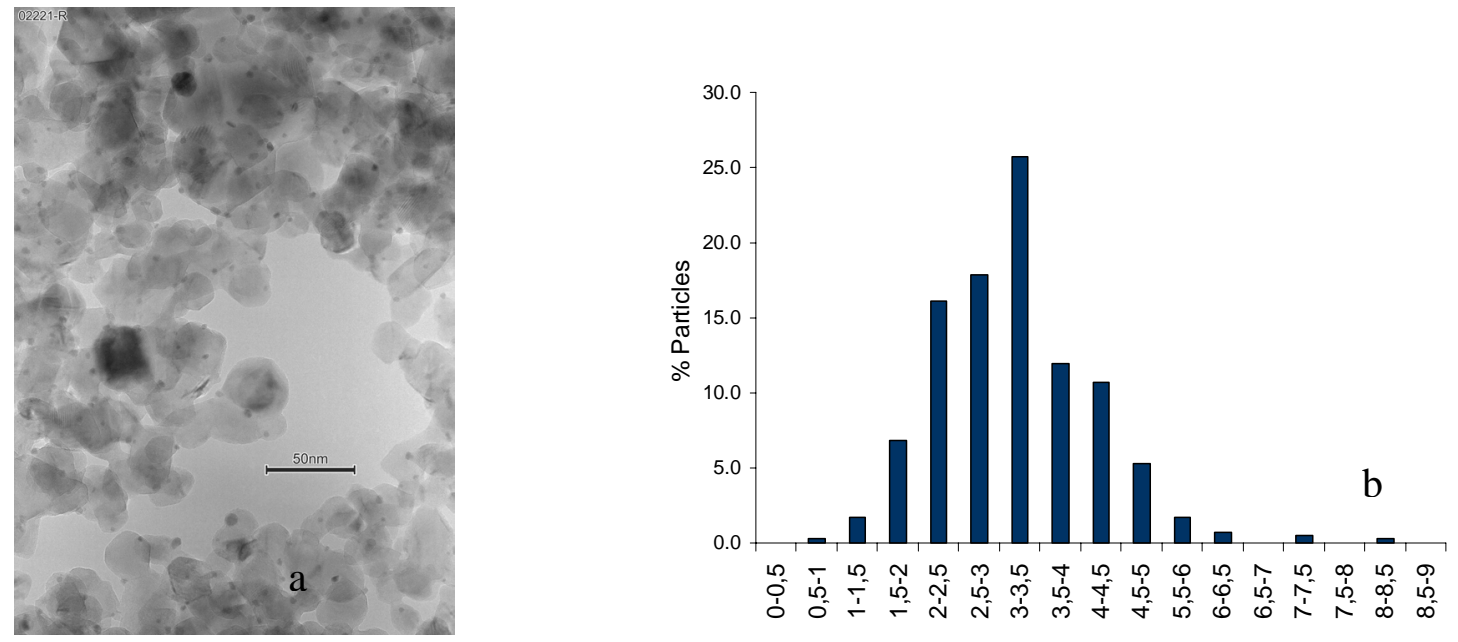

Figure S2. Picture of SPR16 reactor. The system allows to perform up to sixteen reactions in parallel at high pressure levels. Actions such as feeding the reaction mixture, controlling the reaction conditions, and taking samples from each autoclave can be automatically carried out by the equipment.

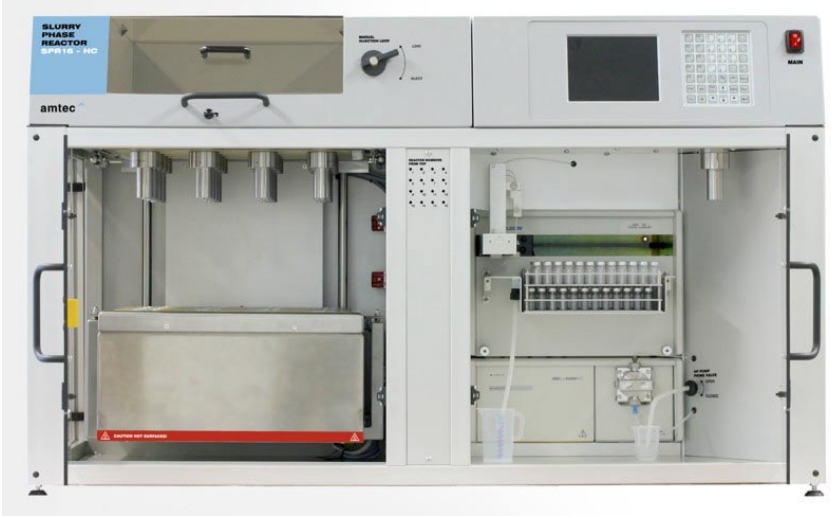

Table S1. Reaction mixture composition for the selected $\alpha, \beta$-unsaturated nitrocompounds.

\begin{tabular}{ccccc}
\hline Substrate & Solvent & mgr Catalyst $^{\text {a }}$ & $\begin{array}{c}\mathbf{w t} \% \\
\text { Substrate }\end{array}$ & $\begin{array}{c}\text { wt\% } \\
\text { Internal Standard }\end{array}$ \\
\hline Trans- $\beta$-Nitrostyrene & Toluene & 25 & 4.8 & 1.1 \\
Trans-4-Methoxy- & Toluene & 30 & 5.7 & 1.1 \\
$\beta-N$-Nitrostyrene & & & & \\
Trans-4-Bromo- & Toluene & 25 & 6.0 & 1.1 \\
$\beta-N$-Nitrostyrene & Toluene & 30 & 5.1 & 1.1 \\
$\beta, 2-$ Dinitrostyrene & Ethanol & 40 & 14.8 & 2.0 \\
1-Nitro-1-Cyclohexene & a & & &
\end{tabular}

